DOI: $10.3901 / J M E .2015 .07 .197$

\title{
磨削过程建模与仿真研究现状*
}

\author{
傅玉灿田霖 徐九华 杨 路 赵家延 \\ (南京航空航天大学机电学院 南京 210016)
}

\begin{abstract}
摘要: 概述磨削过程建模与仿真的国内外研究现状，对基于经验分析、理论解析及有限元分析的三类建模与仿真研究发展历 程与趋势进行综述, 并对各类模型建立方法与仿真结果进行详细论述, 同时深入分析这些模型应用的局限性, 指出建立在有 限试验数据基础上的经验模型预测水平较低; 基于理想假设基础上的理论模型建模过程复杂; 而由于技术问题，有限元法则 仅限于对单颗磨粒磨削过程的仿真, 有鉴于此, 提出基于超硬磨粒有序排布砂轮的磨削过程建模与仿真方法, 并进一步着重 阐述其在高速高效磨削过程预测、优化与控制应用前景及意义。

关键词：磨削过程；建模；仿真；预测；优化

中图分类号: TG580

\section{Development and Application on the Grinding Process Modeling and Simulation}

\author{
FU Yucan TIAN Lin XU Jiuhua YANG Lu ZHAO Jiayan \\ (College of Mechanical and Electrical Engineering, Nanjing University of Aeronautics and Astronautics, \\ Nanjing 210016)
}

\begin{abstract}
The research of the grinding process modeling and simulation is presented. The development progress and its tendency of three types of modeling methods and simulation are described results, which are based on empirical analysis, theoretical analysis and finite element technology. The method of modeling and simulating results are discussed respectively in detail. Meanwhile the limitations of application of models are analyzed. It is indicated that the empirical models are based on finite data of experiments, which have low level of prediction abilities, the theoretical models which are set up on ideal assumption is complexity, and the finite element models are restricted to analyze the process of single-grit grinding due to technical problem, on that account, the methods of grinding process modeling and simulation research based on the regular abrasive distribution grinding wheel are introduced. Furthermore, their present situation prospect and significance for prediction, optimization and control of high speed and high efficiency grinding are elaborated.
\end{abstract}

Key words: grinding process; modeling; simulation; prediction; optimization

\section{0 前言}

磨削过程建模是指在认知基础上抽象地描述 磨削过程中各物理量之间关系; 仿真则是利用上述 各类模型对磨削加工进行模拟的过程。建模与仿真 最终要实现三大应用，即对磨削结果的预测、对磨 削条件的优化及磨削过程的控制。如图 1 所示, 建 模与仿真过程大致分为认知、抽象、验证三个阶段, 首先对磨削机理进行系统的认识; 其次是抽象, 所 谓抽象就是将实际磨削过程通过一定的方法提炼成

* 国家重点基础发展研究计划(2009CB724403)和江苏省普通高校研究生 科研创新计划(CX10B 091Z)资助项目。20140606 收到初稿, 20141125 收到修改稿
物理关系并形成磨削模型，其次是试验，即利用模 型进行磨削过程仿真并与试验结果进行对比，以验 证模型的预测与控制能力，若其能力符合生产实际 需要则为可应用模型, 反之则要再次进行 “认识抽象-验证”，如此循环最终将形成具有一定应用能 力的磨削过程模型。

众所周知，磨削过程异常复杂，以致今日都没 有形成针对磨削过程的全面系统的认识体系, 同时, 新材料，新工具(砂轮、砂带及研磨工具等)以及新 的磨削技术的不断出现对磨削模型的应用能力提出 了更高的要求。总之，如图 1 所示，磨削过程的复 杂性与磨削技术的更新是磨削过程建模与仿真研究 不断发展与革新的原动力。 


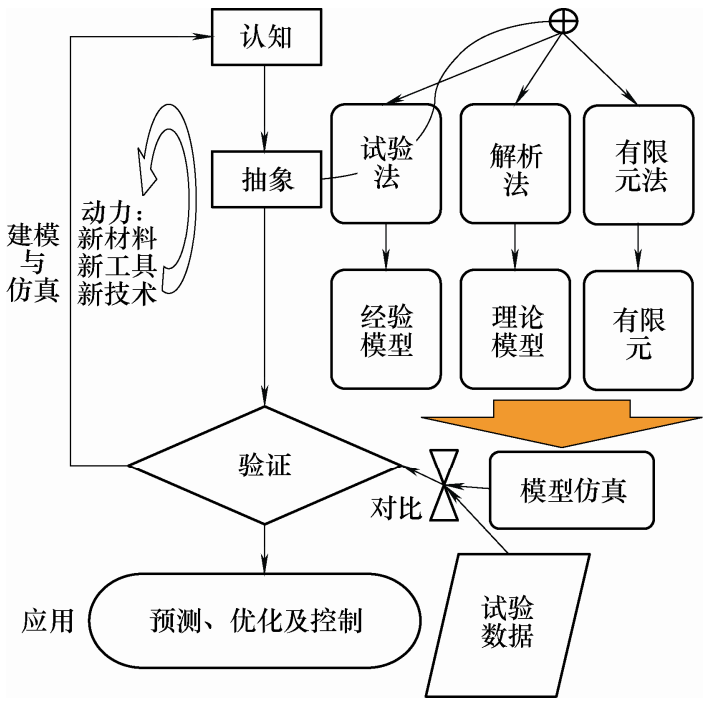

图 1 磨削过程模型建立过程

\section{1 磨削过程建模与仿真}

如图 1 所示, 磨削过程模型按其建模方法分类, 分为经验模型, 理论模型以及仿真模型。即基于试 验建立磨削过程输入输出量关系的经验模型、以解 析为主的理论模型以及利用有限元技术的有限元 模型。

\section{1 经验建模与仿真}

由大量磨削加工经验可知, 磨削条件与磨削 力、磨削温度之间存在着一一对应的必然联系, 诸 如磨削速度的提高会带来磨削力的降低, 磨削深度 与磨削进给速度的提高会带来磨削力的上升, 即当 磨削条件一定，磨削力、磨削温度也就唯一确定了, 这就形成了经验建模的依据。因此, 众多的经验模 型是不以研究磨削过程机理为目的的, 而是以大量 磨削试验结果为基础, 通过数学的方法建立磨削条 件与磨削过程参量之间的函数对应关系。表 1 列举 了几种典型的经验模型分析方法及特点。量纲分析 法 $^{[1-2]}$ 与回归方法 (或方差分析 $)^{[3-4]}$ 是两种最基本的 经验模型建模方法, 前者是通过构造量纲 $Q_{1}$ 与 $Q_{2}$ 建立磨削过程量(磨削力 $F_{t}$ 、磨削温度 $T$ ) 与磨削用 量的关系, 后者是对试验数据进行回归建立磨削过 程量与磨削用量之间的幂指数关系。总之, 回归分 析与量纲分析法虽然能够较准确地预测磨削力 $F_{t}$ 与磨削温度 $T$, 但是利用幂指数函数并不能反映磨 削条件与磨削过程参量之间的复杂联系, 而且针对 同种材料各个研究者给出的指数值差异较大。考虑 到磨削过程影响因素相互作用的复杂性, 将智能化 自学习技术应用于数据处理并形成磨削过程模型的 课题已成为经验模型发展的重要研究方向。如表 1 所示的周会娜 ${ }^{[5]}$ 利用 BP 神经网络与遗传算法对脆
性难加工材料磨削力模型进行深入研究。FUH 等 ${ }^{[6]}$ 则利用神经网络技术成功对缓进给磨削过程中磨削 力进行了预测并解决了全局解的快速收玫问题。灰 色系统理论是一个通过对已知数据进行灰色生成, 寻找系统内在规律，实现对数据进行管理和控制的 新兴理论 ${ }^{[7]}$ 。杨秋平等 ${ }^{[8]}$ 利用灰色模型 $\mathrm{GM}(1,1)$ 对 外圆磨削的磨削力进行了预测，揭示了灰色理论应 用于磨削力建模的有限性, 试验数据分布不能较好 地服从指数 $e$ 分布规律时, 所得灰色模型对建模数 据的模拟或拟合精度会较差。

表 1 经验建模

\begin{tabular}{|c|c|c|}
\hline & 分析方法 & 特点 \\
\hline 文献[1-2] & 量纲分析法 & 小范围预测 \\
\hline 文献[3-4] & 回归方法方差分析 & 预测范围小, 预测准确性较高, \\
\hline \multirow{2}{*}{ 文献[5-7] } & BP & 自学习型, 前期试验量大, 预测 \\
\hline & ANN & 范围广，预测准确度高 \\
\hline 文献[8-9] & 灰色系统理论 & $\begin{array}{l}\text { 可以对不确定特征进行预测, 预 } \\
\text { 测结果是一个区间 }\end{array}$ \\
\hline
\end{tabular}

\section{2 理论建模与仿真}

理论模型的建立是基于对磨削机理深刻认识 之上对磨削过程机制的物理解析, 因此磨削力, 磨 削温度的推导公式都是解析式, 都具有一定的物理 意义。建立的方法一般都是将磨削机制建立磨削过 程参量与磨削条件之间的物理关系，并根据物理关 系建立解析公式，通过试验确定解析式参量。

\subsection{1 砂轮地貌及磨削过程特征建模}

磨削中材料的去除主要是由和车、铣等加工方 法相类似的切屑形成过程完成的，只是磨屑要小得 多, 因此研究磨削运动几何特征是建立磨削理论模 型的基础, 但是对大多数加工过程而言, 刀具与工 件的几何特征和相互作用很好确定，而对磨削却不 易做到。磨削过程是砂轮工作表面上无规则排列的 大量的几何形状不确定的切刃, 与工件材料综合作 用的过程。地貌的随机性决定了砂轮上每一切刃的 切削几何学均不相同。

为分析研究磨削切屑几何学, 磨削建模理论对 砂轮地貌进行了合理假设。

假设条件一：磨粒形状规则，磨粒尖角为雉形 或圆形。

假设条件二：磨粒等高性一致，即磨粒顶点半 径一致。

假设条件三：磨粒排布均匀，即磨粒分布密度 $C$ 为定值或参与切削的磨粒周向间距 $p$ 均为定值。

在此三大假设基础上国内外学者在砂轮地貌 与磨削过程特征参数建模方面做了大量工作，这些 工作主要集中在对有效磨粒数、磨削弧长以及单颗 
磨粒切屑厚度三个重要参数的建模, 西北工业大学 任敬心等 ${ }^{[3]}$ 利用砂轮表面磨粒分布密度 $C$ 参数建立 了静态有效磨粒数 $N_{s t}$ 公式, 然而磨削过程是动态 过程, 该公式未能反映这一事实, 因此 WERNER ${ }^{[10]}$ 根据砂轮运动特征结合分布密度参数建立了与磨削 用量有关的动态有效磨粒数 $N_{d}$ 模型; 通过进一步试 验 YEGENOGLU ${ }^{[1]}$ 发现了磨粒出露高度因素对有 效磨粒数的影响机制, 并将该机制引入公式中。由 此可见, 有效磨粒数模型的建立过程经历由静态到 动态, 由简单到复杂的过程, 预测能力却逐渐增强, 究其原因是磨削过程的复杂性所决定的, 考虑因素 越多, 理论与实际就越一致。磨削弧长、当量切厚 以及单颗磨粒切屑厚度建模也经历了类似的过程, 任敬心等 ${ }^{[3]}$, 刘镇昌 ${ }^{[12]} 、$ LINDSAY 等 ${ }^{[13]} 、$ KUMAR 等 ${ }^{[14]}$ 以及 $\mathrm{QI}$ 等 ${ }^{[15]}$ 等分别从几何特征、磨削方式、 砂轮变形以及表面粗䊅度等不同因素对磨削弧长的 公式进行了各自的诠释, 逐步完善了磨削弧长的数 学模型。KURREIN ${ }^{[16]} 、$ COLDING $^{[17]}$ 以及 PETERS ${ }^{[18]}$ 在对等厚薄片成屑假设基础上建立了当量切厚 $a_{e q}$ 模型, 虽然在一定程度上解释了磨削用量三要素 $v_{s}$ 、 $v_{w} 、 a_{p}$ 与磨削成屑机制之间的关系, 但并未将砂轮 地貌纳入参考因素。因此众多学者提出了单颗磨粒 切屑厚度 $a_{g}$ 模型, WERNER 等 ${ }^{[10,19]}$ 以磨粒分布密 度和砂轮有效磨粒数建立了单颗磨粒切屑厚度模 型, 而 PAHLITZSCH 等 ${ }^{[20-21]}$ 则以磨粒间距建立了 $a_{g}$ 模型, 单颗磨粒切屑厚度模型是最重要的磨削过 程特征模型, 是建立磨削力、磨削温度模型的基础, 并与砂轮地貌模型息息相关。

\subsection{2 磨削力模型}

磨削力是对磨削过程描述的最重要的物理量。 MERCHAT $^{[22]}$ 在 1945 年推导出经典成屑理论模型 并提出 “尺寸效应” 机理后, 为磨削力建模提供了 重要的理论支持。如表 2 所示, 经典磨削力模型的 物理意义: 磨削力 $F$ 等于磨削弧区内所有磨粒所受 力的总和, 即单颗磨粒磨削力 $F_{g}$ 与磨粒数 $N_{d}$ 的乘 积, 而单颗磨粒磨削力则是单位磨削力 $K$ 与磨粒切屑接触面积 $A$ 的乘积。根据这一基本物理模型, 以运动几何学为基础, 日本的与臼井英治 ${ }^{[1]}$ 假设磨 粒为圆雉形, 以切削方向角 $\Psi$ 为积分变量建立了磨 削力模型的初级形式(表 2)。考虑到磨削弧长上各点 的单颗磨粒切屑厚度与有效磨粒数随机性与可变 性, WERNER ${ }^{[23]}$ 建立以可变弧长 $i_{s}$ 为积分变量的 磨削力模型, 由表 2 可知, 该模型中存在一指数 $n_{g}$, 它反映了切削变形力与摩擦力之间的关系, 然而该 模型并未从物理意义上将上述二力加以区分, 因此 湖南大学的 LI 等 ${ }^{[24]}$ 对该模型做了进一步分析, 直 观地划分为切屑变形力 $F_{t s}$ 与摩擦力 $F_{t c}$ 两部分, 表 2 中 $\bar{\delta}$ 为单颗磨粒的平均磨钝顶面积, $\chi$ 为磨粒圆 雉定焦半角, $\bar{p}$ 则为磨刃与工件接触平均压强, $\omega$ 为砂轮旋转角速度。

\section{表 2 磨削力理论建模}

\begin{tabular}{|c|c|c|c|c|}
\hline & \multicolumn{3}{|c|}{ 基本公式 } & \multirow{2}{*}{ 考虑因素 } \\
\hline & 磨削力 $F$ & 单颗磨粒切屑-工件接触面积 $A$ & 弧区内磨粒有效数 $N_{d}$ & \\
\hline 文献[1] & $\int_{-\frac{\pi}{2}}^{\frac{\pi}{2}} K A N_{\mathrm{d}} \mathrm{d} \psi$ & $\frac{1}{2} A_{g}^{2} \sin \chi \cos ^{2} \psi$ & $\frac{d_{s}(a+\delta) f_{a}}{2 \omega^{2}}$ & 磨粒几何形状 \\
\hline 文献[23] & $F=\int_{0}^{l_{s}} K A N_{\mathrm{d}} \mathrm{d} l_{s}$ & $\left(A_{g} C^{-\beta}\left(\frac{v_{w}}{v_{s}}\right)^{1-\alpha}\left(\frac{a_{p}}{d_{s}}\right)^{1--\frac{\alpha}{2}}\left(\frac{l_{s}}{l_{s}}\right)^{1-\alpha}\right)^{n g}$ & $A_{g} C^{\beta}\left(\frac{v_{w}}{v_{s}}\right)^{\alpha}\left(\frac{a_{p}}{d_{s}}\right)^{\alpha / 2}\left(\frac{i_{s}}{l_{s}}\right)^{\alpha}$ & $\begin{array}{l}\text { 砂轮表面磨 } \\
\text { 粒分布密度 } C \\
\text { 与有效磨粒 } \\
\text { 数的可变性 }\end{array}$ \\
\hline 文献[24] & $\begin{array}{l}K A \\
\int_{0}^{l_{s}} K A N_{\mathrm{d}} \mathrm{d} l_{s}^{\prime}\end{array}$ & $\begin{array}{l}\sum A=\frac{z_{q}}{v_{s}}=\frac{v_{w} a_{p}}{v_{s}} \\
\bar{\delta} \bar{p}\end{array}$ & $A_{g} C^{\beta}\left(\frac{v_{w}}{v_{s}}\right)^{\alpha}\left(\frac{a_{p}}{d_{s}}\right)^{\alpha / 2}\left(\frac{l_{s}}{l_{s}}\right)^{\alpha}$ & $\begin{array}{l}\text { 考虑将切屑变形 } \\
\text { 力与摩擦力区别 } \\
\text { 分析 }\end{array}$ \\
\hline
\end{tabular}

随着磨削技术的不断发展，尤其是高速高效磨 削技术的出现, 使得高速磨削理论研究者们逐渐认 识到, 高速磨削加工机理不同于普通磨削加工, 经 典磨削力模型应用能力下降。高速磨削时, 磨粒高 速冲击作用表层材料, 单个磨屑的形成时间很短, 即在极短的时间内表层材料经历了高应变率、高应 变和高温变化而形成磨屑, 因此高速磨屑变形机研
究在高速磨削力建模中具有重要的意义。湖南大学 金滩等 ${ }^{[25]}$ 在这方面进行了卓有成效的探索, 认为传 统磨削机制的建立都是基于材料准静态变形行为 的, 忽略了应变率效应对材料变形行为的影响, 并 建立高速磨削条件下的应变与应变率公式; 中南大 学陈勇平等 ${ }^{[26]}$ 在金滩模型基础上, 以磨削能为纽带 建立了应变率与磨削力之间的关系, 并对磨削力进 


\section{行了预测。}

磨削过程的影响因素繁多, 如前所述, 通常用 若干主要影响因素的指数形式建立模型来描述磨削 结果的规律, 这虽能用于一般情况所需要的对磨削 结果的估算，但许多未考虑的实际因素影响的规律， 又会使实际数据与计算值相差较大, 而且由某一个 特定试验所得的结果往往不能转换在另一过程应 用, 致使试验工作量繁重, 不利于各种程序规律的 掌握与控制, 为了弥补这些不足, 目前有些学者致 力于用 “计算机+理论模型” 的方法来模拟磨削过 程, 建立动态砂轮地貌模型, 完成动态磨削过程的 仿真。如德国 Kaiserslautern 大学 AURICH 等 ${ }^{[27}$ 则 对随机排布砂轮进行实测, 建立三维模型如图 2 所 示, 根据几何运动仿真可获得砂轮表面每颗磨粒未 变形切削面积 $A_{c u}$, 砂轮上所有磨粒的未变形切削面 积与单位磨削力 $K$ 的乘积之和即为动态磨削力, 仿 真计算结果如图 2 所示, 这一建模原理与表 2 中所 述磨削力类似, 唯一不同的该磨削力是建立在实测 的动态砂轮地貌模型基础上的, 因此具有该磨削力 模型也就具有动态特性。HUANG ${ }^{[28]}$ 则通过类似信 号频率密度谱分析的方法对砂轮地貌进行了模拟, 建立了砂轮磨粒分布密度与砂轮旋转角度 $\beta$ 之间的 动态关系(图 3b)建立了随砂轮密度变化的动态磨削 力 $f_{y}$ 模型如图 $3 \mathrm{c}$ 所示。
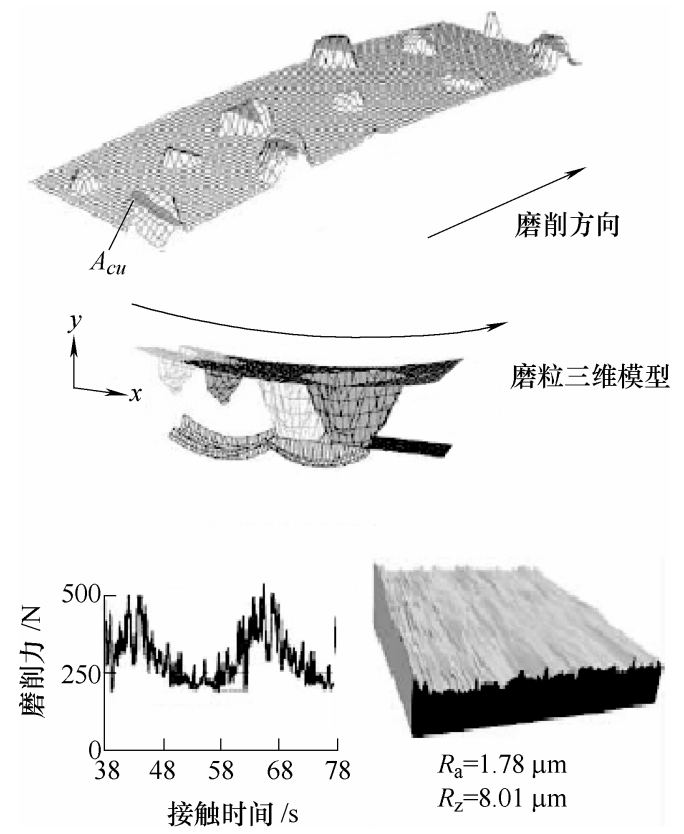

图 2 基于三维砂轮地貌的动态磨削力模型

\subsection{3 磨削温度模型}

磨削过程由于磨削力的存在产生了磨削能量 又称磨削比能, 这些能量除一部分用于切屑变形外, 大部分转变为热量并传入工件, 根据传热学理论, 磨削弧区的热量按照一定规律的热流密度分布模型

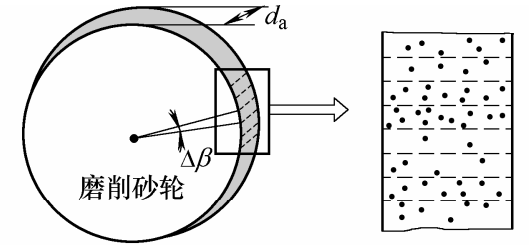

(a) 砂轮形貌模型

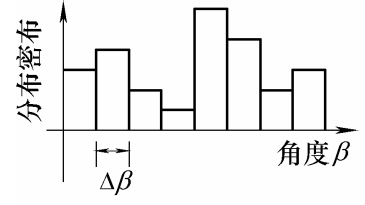

(b) 分布密度模型

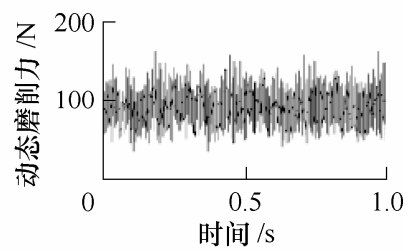

(c) 仿真结果
图 3 基于砂轮磨粒分布密度变化的动态磨削力模型

传入工件, 并以工件进给速度在磨削工件表面移动, 使得工件升温。这就是著名的 JAEGER ${ }^{[29]}$ 移动热源 温度场理论。移动热源模型主要解决的两个问题是 热比例分配系数的确定与热源分布模型的建立。模 型热量分配比例的计算一直是一个十分复杂的问 题，由于其涉及工件材料传热特性、砂轮传热特性、 磨削参数以及冷却状态等因素的综合影响, 因此尚 无一个十分完善的理论来确定传入工件的热量比 例, 而且缓进给磨削中由于成膜沸腾的存在, 其冷 却状态是最不确定的参量, 表 3 列举了两种典型的 热比例分配系数 $R$ 的计算模型。OUTWATER 等 ${ }^{[30]}$ 从分析磨削过程的单颗磨粒与工件以及切屑之间微 观区域的传热方式角度建立热比例分配关系，该模 型开创了理论解析磨削热量分配关系的先例, 然而 该模型却忽略了冷却液带走热量, 因此需要不断对 参数进行修正以符合实际试验结果; ROWE 等 ${ }^{[31]}$ 则将砂轮与磨削液视为复合体, 认为磨削液是砂轮 的一部分，建立的模型如表 3 所示。目前为止，热 比例分配模型的预测能力很有限, 其中一个原因就 是磨削液带走的热量在磨削过程中是不确定的, 如 缓磨中，磨削液正常状态下带走的热量与膜沸腾状 态带走的热量完全不同; 同时磨削液带走热量的比 例的影响机制不明确, 如砂轮线速度的增加导致砂 轮表面存在气障，磨削液不易进入弧区，从而降低 了磨削液带走的热量, 然而这种影响关系至今也未 能明确。表 3 中 $r_{0}$ 为磨刃切削宽度与磨刃切削深度 的比值, $c$ 为热容, $k$ 和 $k_{g}$ 分别为工件传热系数和 砂轮传热系数。

表 3 热比例分配系数标准模型

\begin{tabular}{lll}
\hline & 热比例分配系数标准模型 $R$ & \multicolumn{1}{c}{ 考虑因素 } \\
\hline 文献[30] & $\left(1+\left(k_{g} / r_{0} v_{w}(k \rho c)_{w}\right)^{0.5}\right)^{-1}$ & $\begin{array}{l}\text { 单颗磨粒-工件接触 } \\
\text { 传热方式 }\end{array}$ \\
\hline 文献[31] & $\left(1+\left(v_{s}(k \rho c)_{s} / v_{w}(k \rho c)_{w}\right)^{0.5}\right)^{-1}$ & 砂轮与磨削液复合体 \\
\hline
\end{tabular}


如图 4 所示, 张否 ${ }^{\left[{ }^{[} 2\right]}$ 对不同热源分布模型所计 算得到的表面温度作对比研究发现, 不同热源分布 模型对工件表面最高温度所在的位置是有一定影 响, 但对表面最高温度数值的影响却很小。随着技 术的发展, 缓进磨开槽砂轮的出现对基于磨粒连续 分布砂轮基础上建立的传统热源分布模型提出新的 要求, 为此, 南京航空航天大学傅玉灿等 ${ }^{[33]}$ 对开槽 砂轮断续磨削的温度场进行分析, 提出采用热源温 度场叠加法(简称热源法)来解析缓进给深切磨削时 的温度场, 建立了温度场模型; 李迎 ${ }^{[34]}$ 则引入信 号卷积概念对考虑成膜沸腾热源模型进行了数学 描述。

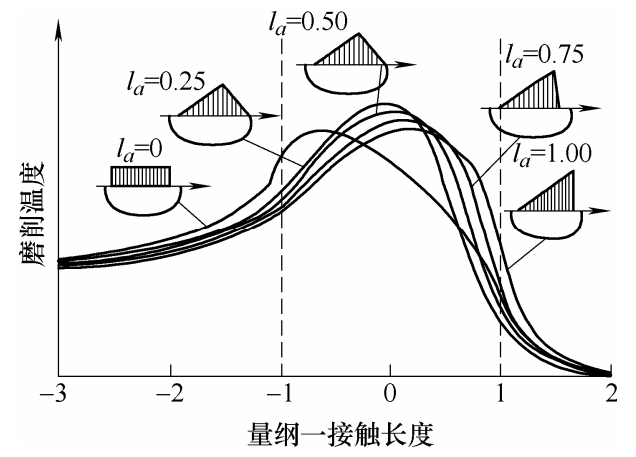

图 4 不同热源模型的表面温度分布

\section{3 有限元建模与仿真}

磨削过程的复杂性使得磨削存在错综复杂的 磨削机理关系, 建立磨削条件与磨削结果之间的物 理模型就要综合考虑各种影响, 其中各因素之间的 耦合关系是通过简单的数学公式是无法建立的, 由 于磨削加工的数值模拟方法可综合考虑多种磨削参 数的作用, 并能有效预测磨削过程的各种现象, 数 值模拟方法(如有限元模型(Finitie element method, FEM)和分子动力学模型(Molecular dynamics, MD)) 已成为当今研究磨削加工的重要手段。

\subsection{1 有限元(FEM)模型}

磨削加工在微观尺度的有限元模拟一般主要 研究单磨粒的工作过程。众所周知, 磨削过程单颗 磨粒切刃呈负前角, 但是 OHBUCHI 等 ${ }^{[35]}$ 则通过试 验发现了磨削死区的存在单颗成屑过程中, 认为由 于材料在负前角处堆积, 类似于车削的粘刀现象, 因此他建立的磨粒模型前刀面上突起一块(表 4)从 而形成正前角, 并对该理论进行了细致的仿真研究。 WARNECK 等 ${ }^{[36]}$ 则对磨削过程宏观和微观运动进 行分析, 建立了三维 FEM 磨削模型, 并综合运用 理论解析法搭建磨削过程预测系统。

\subsection{2 分子动力学 $(\mathrm{MD})$ 模型}

分子动力模型直接根据材料的微观晶体结构 建模, MD 模型中分子之间的相互作用由经验势能
函数决定, $\mathrm{MD}$ 模拟系统的演进则根据经典力学原 理, 系统物理状态则根据统计热力学物理原理来得 到。分子动力学方法的研究源于 1950 年 ALDER 等 ${ }^{[38-39]}$ 的工作, 分子动力模拟应用于材料切削和磨 削加工的研究最早开始于 20 世纪 80 年代末。 BELAK 等 ${ }^{[40]}$ 研究了金刚石在金属平面上的刻划及 压痕试验; 模拟结果发现在弹性变形阶段, 原子级 的应力场和宏观尺度试验结果相似, 但发生塑性变 形时, 分子模拟结果的原子级的屈服应力远高于宏 观试验结果。SHIMIZU 等 ${ }^{[41]}$ 利用这个原理, 研究 了 $50 \mathrm{~m} / \mathrm{s}$ 到 $3000 \mathrm{~m} / \mathrm{s}$ 极限速度下的磨削过程分子 动力学仿真模型仍受到很大的限制, 一般只用于分 析单个磨粒的工作过程, 表 4 列举了三个典型的 MD 单颗磨粒仿真模型, KOMANDURI 等 ${ }^{[2-43]}$ 都是 采用 $2 \mathrm{D}$ 模型的, 不同的是前者将磨粒简化为锥形, 后者则认为磨粒尖角为圆形。 RENTSCH 等 ${ }^{[44]}$ 则通 过建立3D模型进行单颗磨粒切削过程仿真研究的。

表 4 有限元模型

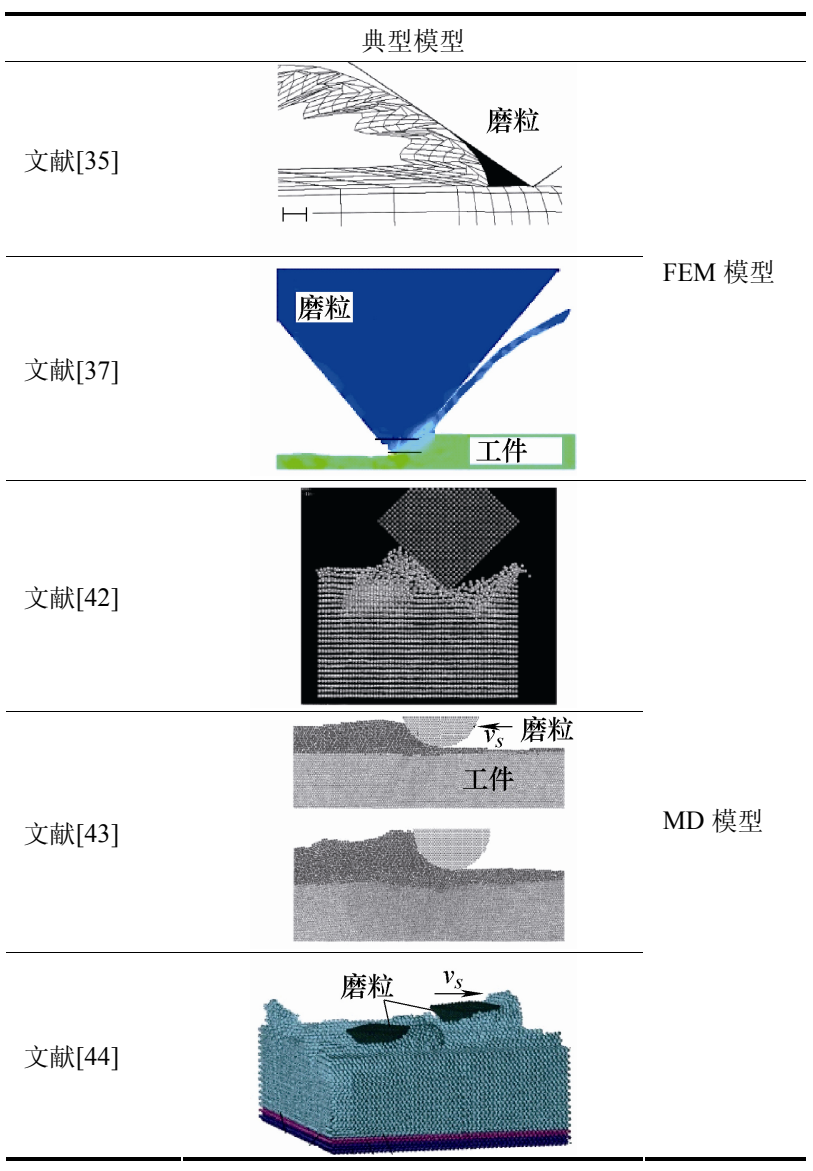

2 磨削过程建模与仿真应用中存在的问题

\section{1 经验建模与仿真研究存在问题}

实际加工过程中, 大部分都是利用经验模型对 磨削工艺进行优化，从而获得最佳用量组合。然而 
经验模型的建立依赖大量的试验数据, 建模成本很 高, 而且预测准确性取决于样本空间的大小，同时 纵观经验模型都未曾考虑砂轮地貌这一重要磨削条 件对磨削结果的影响, 因此应用能力有限。

\section{2 理论建模与仿真研究存在问题}

理论模型的建立试验成本较低, 对磨削过程能 够很好地进行模拟, 但由于其预测准确度受基于实 测和假设基础上建立的砂轮地貌模型限制, 同时基 于砂轮地貌三大假设基础上的理论模型对磨削工艺 的制定具有重要的有意义的参考价值, 但在实际应 用中由于建模过程复杂仅限于对磨削结果及磨削规 律的定性分析。

\section{3 有限元建模与仿真研究存在问题}

有限元法中, 单元划分数量的多少在很大程度 上决定了数值模型求解的计算复杂度。磨削加工是 由大量的磨粒作用于工件的整体切削过程, 若对所 有磨粒都离散单元化, 则模型变得复杂而且单元数 量过多, 模型的求解需要庞大的计算量。另一方面, 只有对有限元模型进行必要修正(包括本构关系修 正、网格划分修正等)才可以实现对已有试验结果的 拟合，因而从本质上决定了有限元模型对当前试验 结果的依赖性, 预测能力自然会有所下降。而分子 动力学仿真技术应用于磨削过程建模还处于探索阶 段, 并未成熟, 因此一般只用于分析单个磨粒的工 作过程。

\section{3 基于超硬磨粒有序排布砂轮的高速 磨削过程建模}

磨削建模与仿真旨在实现对磨削过程的控制 与预测, 然而传统的砂轮制作工艺(陶瓷烧结, 电镀 等工艺)决定了砂轮表面磨粒的无序性, 即地貌在空 间与时域上的无序性造成了磨削过程的复杂性, 正 如文献[45]所称, 砂轮形貌的复杂与冷却液供给的 动态特性决定了磨削过程是一个动态过程, 并最终 导致磨削结果变得不可预测。因此迫切需要超硬磨 料单层有序排布砂轮以满足理论模型中关于地貌建 模的三大假设条件, 从而实现对磨削过程的可控, 同时随着高速磨削技术的发展及在航空类难加工材 料高效加工中的广泛应用, 使得普通磨削速度条件 下建立的准静态磨削过程模型对实际生产工艺的指 导作用的地位面临新的挑战, 为此, 南京航空航天 大学进行了长期深入的研究 ${ }^{[46]}$, 提出通过研制超硬 磨粒有序排布砂轮来达到了降低磨削过程复杂性的 目的, 并据此建立了有序排布砂轮地貌模型, 对磨
削过程进行了建模与仿真的从而提高了磨削模型预 测能力的研究思路, 进一步发挥了难加工材料高速 高效磨削潜力。基于超硬磨粒有序排布砂轮的高速 磨削过程建模研究的具体流程如图 5 所示, 首先通 过创新设计单颗磨粒高速磨削试验方法 ${ }^{[47]}$, 获得了 与实际磨削相同厚度的磨屑, 对高速条件下临界成 屑厚度进行了定量分析，研究了高速单颗磨粒成屑 机制, 南京航空航天大学田霖等 ${ }^{[48-49]}$ 在此基础上提 出了 “速度效应” 理论, 即保持单颗磨粒切厚恒定 条件下，提高磨削速度的同时提高进给速度可以达 到增加去除率且不降低表面质量的效果。因此利用 磨粒钎焊技术实现磨粒的有序排布并达到单颗磨粒 切厚均匀一致成为图 5 所示流程的关键环节，即建 立砂轮地貌特征要素(图 6 所示排布角度 $\alpha$, 排布轴 向 $\Delta x$ 与周向间距 $\Delta z$ 等)并以此建立了有序排布砂轮 地貌模型。

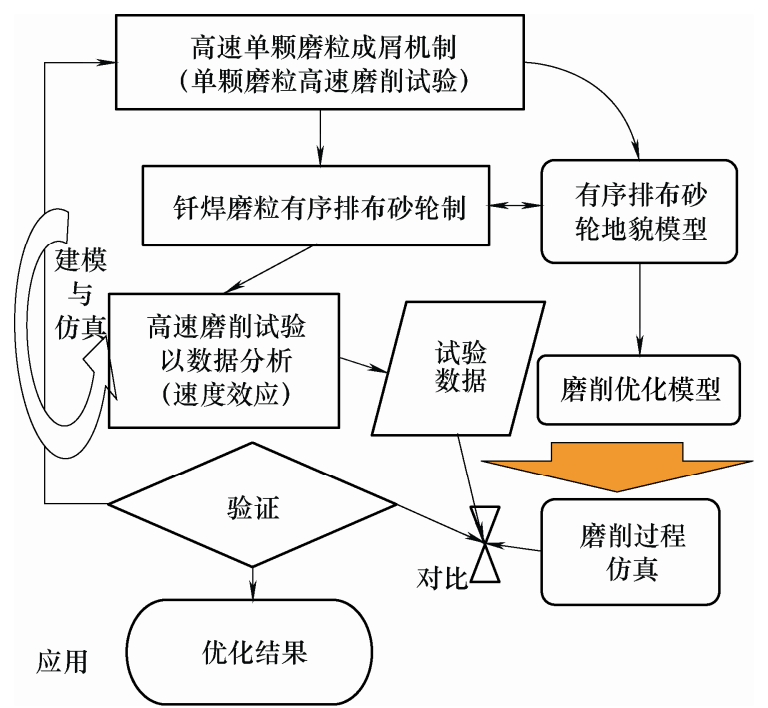

图 5 基于磨粒有序排布砂轮的磨削过程建模流程
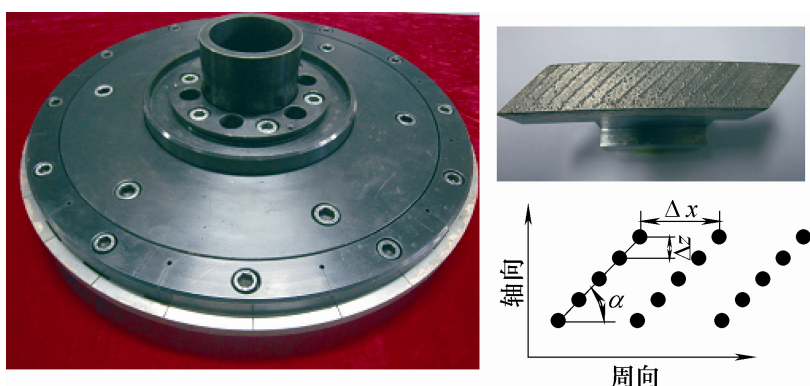

图 6 单层钎焊磨粒有序排布砂轮地貌

在此理论指导下，利用有序排布砂轮高速磨削 试验所获得的工艺数据, 对单颗磨粒切屑厚度、材 料特性以及磨削速度三因素与磨削过程的四方面 (成屑机制、磨削力、磨削比能及磨削温度)之间相 互作用关系进行了细致研究, 并根据按照加工要求 和用量条件, 以磨削比能 $e_{s}$ 最小为目标和以表面粗 
粘度 $R_{\mathrm{a} 0}$ 、容屑空间 $C_{s}$ 、弧区平均热流密度 $q_{\text {lim }}$ 、极 限磨削温度 $T_{l i m}$ 以及磨削参数范围为约束条件设计 砂轮地貌和优化磨削工艺的创新思想, 建立了如表 5 所示磨削优化系统模型 ${ }^{[50]}$, 该模型中存在众多与 被加工材料加工特性以及砂轮地貌有关的参数, 因 此如图 5 所示, 利用钎焊有序排布砂轮对被加工材 料进行高速磨削试验, 通过数据分析可以获得参数, 同时也对被加工材料的速度效应规律进行了认知, 在此基础上就可以通过利用有序排布砂轮对该材料 进行有效的高速磨削工艺及砂轮地貌的优化。因此, 基于磨粒有序排布砂轮的磨削过程建模与仿真方法 与研究思路为新材料尤其是难加工材料的高速高效 磨削过程预测与控制提供了重要的研究方向, 具有 重要的现实指导意义。

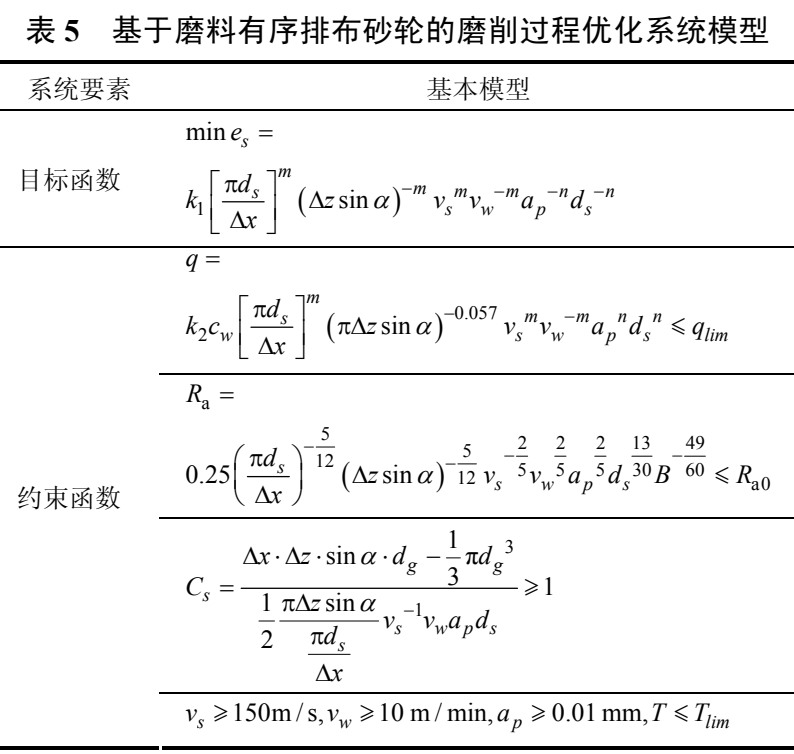

\section{4 结论}

理论模型与有限元模型的结合实现动态磨削 力的模拟、分子动力学仿真的应用以及有序排布砂 轮的实现都为磨削过程建模与仿真工作提供了很好 研究方向, 总之, 建模与仿真最终是要实现应用的, 提高应用能力, 就必须不断利用新的建模方法、新 工具来完善磨削过程模型, 以符合磨削过程的一般 规律, 这样才能够实现对磨削结果的预测, 磨削工 艺优化以及磨削过程的控制, 进一步提高磨削加工 效率。

\section{参 考 文 献}

[1] 白井英治. 切削磨削加工学[M]. 高希正, 译. 北京: 机 械工业出版社, 1980.

EIJIU. Studying on cutting and grinding[M]. Translated by GAO Xizheng. Beijing: China Machine Press, 1980.

[2] 张幼桢. 金属切削理论 [M]. 北京: 航空工业出版社, 1987.

ZHANG Youzhen. Metal cutting theory[M]. Beijing: Aviation Industry Press, 1987

[3] 任敬心, 康仁科, 史兴宽. 难加工材料的磨削 [M]. 北 京: 国防工业出版社, 1992.

REN Jingxin, KANG Rrenke, SHI Xingkuan. Grinding technology of difficult-to-machine materials[M]. Beijing: National Defence Industry Press, 1992.

[4] 李伯民, 赵波. 现代磨削技术[M]. 北京：机械工业出 版社, 2003.

LI Bomin, ZHAO Bo. Modern grinding technology[M]. Beijing: China Machine Press, 2003.

[5] 周会娜. 基于 BP 神经网络和遗传算法对工程陶瓷磨削 力建模的研究[D]. 天津: 天津大学, 2007.

ZHOU Huina. Research on the modeling of ceramic grinding force based on the BP neural networks and genetic algorithms[D]. Tianjin: Tianjin University, 2007.

[6] FUH K H, WANG S B. Force modeling and forecasting in creep feed grinding using improved BP neural network[J]. International Journal of Machine Tools and Manufacture, 1997, 37(8): 1167-1172.

[7] MAKSOUD T M A, ATIA M R. Applications of artificial intelligence to grinding operations via neural networks [J]. Machining Science and Technology, 2003, 7(3): 361-387.

[8] 杨秋平, 王卫平. 磨削力灰色建模的有限性研究 [J]. 组 合机床与自动化加工技术，2002(12): 6-8.

YANG Qiuping, WANG Weiping. A study of limitation of grey modeling for grinding forces[J]. Modular Machine Tool \& Automatic Manufacturing Technique, 2002(12): 6-8.

[9] 仇君, 梁式, 庞庭阶. 磨削力的灰色预测模型[J]. 广西 大学学报, 1997(2): 155-157.

QIU Jun, LIANG Shi, PANG Tingjie. The grey modeling for grinding forces[J]. Journal of Guangxi University, 1997(2): 155-157.

[10] WERNER G. Kinematic and mechanic during grinding processes[D]. Aachen, Germany: RWTH, 1971.

[11] YEGENOGLU K. Calculation of characteristic rough topography on the interpretation of $\mathrm{CBN}$ grinding processes [D]. Aachen, Germany: RWTH, 1986.

[12] 刘镇昌. 平面磨削几何接触长度的新公式 [J]. 华中科 技大学学报, 1983(4): 109-111.

LIU Zhenchang. A new formula for geometric length of contact in surface grinding[J]. Journal of Huazhong University of Science and Technology, 1983(4): 109-111.

[13] LINDSAY R P, HAHN R S. On the basic relationships between grinding parameters $[\mathrm{J}]$. Annals of the CIRP, 
1971, 20(1) : 657-660.

[14] KUMAR K V, SHAW M C. The role of wheel-work deflection in grinding operations [J]. ASME, 1981, 103, 73-78.

[15] QI H S, ROWE W B, MILLS B. Experimental investigation of contact behavior in grinding [J]. Tribology International, 1997, 30(4), 283-297.

[16] KURREIN M. The workability of the metals in relation to the Strength test [J]. Workshop Technology, 1927, 21, 612-621.

[17] COLDING B N. A wear Relationship for turning \& milling and grinding-machining economics[D]. Stockholm, Sweden: Stockholm University, 1959.

[18] PETERS J. Current research in the area ad Rectification [J]. Annals of the CIRP, 1967, 15(1): 21-33.

[19] SHAW M C, REICHENBACH G S. The role of chip thickness in grinding $[\mathrm{J}]$. Trans. ASME , 1956, 18: 847-850.

[20] PAHLITZSCH G, HELMERDIG H. Determination and significance of chip thickness in grinding $[\mathrm{J}]$. Workshop Technology, 1943, 11(12): 397-401.

[21] CHEN C, JUNG Y, INASAKI I. Cylindrical and internal grinding of advanced ceramics-grinding fundamentals and applications[J]. Trans. ASME, 1989，39: 201-211.

[22] MALKIN S. 磨削技术理论与应用[M]. 蔡光启, 译. 沈 阳: 东北大学出版社, 2002 .

MALKIN S. Grinding technology theory and applications of machining with abrasives[M]. Translated by CAI Guangqi. Shenyang: Northeastern University Press, 2002.

[23] WERNER G. Influence of work material on grinding forces [J]. Annals of the CIRP, 1978, 27: 243-248.

[24] LI Lijun, FU Jizai. A study of grinding force mathematical model[J]. Annals of the CIRP, 1980: 245-249.

[25] 金滩, 蔡光起. 材料的应变率强化与磨削加工中的尺寸 效应[J]．中国机械工程，1999，10(12)，1401-1417. JIN Tan, CAI Guangqi. Strain rate strengthening of workpiece material and the size effect involved in grinding[J]. China Mechanical Engineering, 1999, 10(12): 1401-1407.

[26] 陈勇平, 唐进元. 磨削加工中的尺寸效应机理研究 [J] . 中国机械工程, 2007, 18(17): 2033-2036.

CHEN Yongping, TANG Jinyuan. Research on the size effect involved in grinding[J]. China Mechanical Engineering, 2007, 18(17): 2033-2036.

[27] AURICH J C, BRAUN O, WARNECKE G. Development of a super abrasive grinding wheel with defined grain structure using kinematic simulation[J]. Annals of the
CIRP, 1991, 12: 327-333.

[28] HUANG Chengchang. A stochastic grinding force model considering random[J]. International Journal of Machine Tools and Manufacture, 2008, 48: 1335-1344.

[29] JAEGER J. Moving sources of heat and the temperature at sliding contacts $[\mathrm{J}]$. Proceedings of the Royal Society of New South Wales, 1942, 76: 203-224.

[30] OUTWATER J O, SHAW M C. Surface temperatures in grinding[J]. Trans. ASME, 1952, 74: 73-78.

[31] ROWE W B, BLACK S, MILLS B, et al. Grinding temperatures and energy partitioning $[\mathrm{J}]$. Proceedings of the Royal Society, 1997, 453(1): 1083-1104.

[32] 张磊. 单程平面磨削淬硬技术的理论分析和试验研究 [D]. 济南: 山东大学, 2006 .

ZHANG Lei. Study on mechanism and experiment of single pass surface grind hardening technology[D]. Jinan: Shandong University, 2006.

[33] 傅玉灿, 徐鸿钧. 开槽砂轮缓进给深切磨削时工件表层 温度场解析 [J]. 中国机械工程, 2002, 7(13): 541-543. FU Yucan, XU Hongjun. Analysis on the temperature field on workpiece surface layer in creep feed grinding with slotted grinding wheel[J]. China Mechanical Engineering, 2002, 7(13): 541-543.

[34] 李迎. 关于难加工材料高效磨削时的热效应与弧区换 热机理的基础研究[D]. 南京: 南京航空航天大学, 1995 . LI Ying. Fundamental studies on the thermal effect and the heat transfer of the high efficiency grinding for difficult-to-machine materials[D]. Nanjing : Nanjing University of Aeronautics and Astronautics, 1995.

[35] OHBUCHI Y, TOBIKAWA. Finite element modeling of chip formation in the domain of negative rake angle cutting $[\mathrm{J}]$. Journal of Engineering Materials and Technology, 2003, 125: 324-332.

[36] WARNECK G, ZITT U. Kinematic simulation for analyzing and predicting high-performance grinding processes[J]. Annals of the CIRP, 1998, 47(1): 265-270.

[37] LAN Yan, LI Xuekun, WALEED B R, et al. Research on microscopic grain workpiece interaction in grinding through micro-cutting simulation[J]. Advanced Materials Research, 2009, 76: 9-14.

[38] ALDER B J. WAINWRIGHT T E. Studies in molecular dynamics. I. General method[J]. The Journal of Chemical Physics, 1959, 31(2): 459-466.

[39] ALDER B J, WAINWRIGHT T E. Studies in molecular dynamics. II. Behavior of a small number of elastic spheres[J]. The Journal of Chemical Physics, 1960, 33(5): 1439-1451. 
[40] BELAK J, STOWERS I F. The indentation and scraping of a metal surface: A molecular dynamics study[J]. ASI Series E, 1991, 220: 511-520.

[41] SHIMIZU J, ZHOU L, EDA H. Molecular dynamics simulation of material removal mechanism beyond propagation speed of plastic wave[J]. LEM JSME, 2003, 21: 309-314.

[42] KOMANDURI R, CHANDRASEKARAN N, RAFF L M. MD simulation of indentation and scratching of single crystal aluminum[J]. Wear, 2000, 240: 113-143.

[43] SHIMIZU J, ZHOU L B, EDA H. Simulation and experimental analysis of super high-speed grinding of ductile material[J]. Journal of Materials Processing Technology, 2002, 129: 19-24.

[44] RENTSCH R, BRINKSMEIER E. Teratology aspects in state of the art MD cutting simulations[C]//Chemnitz in Germany, 8th. CIRP Int. Workshop on Modeling of Machining Operations, 2005: 401-408.

[45] BRINKSMEIER E, AURICH J C, GOVEKAR E, et al. Advances in modeling and simulation of grinding processes[J]. Annals of CIRP, 2006, 55(2): 667-696.

[46] 傅玉灿. 关于进一步开发高效磨削潜力的基础研究 [D]. 南京: 南京航空航天大学, 1999 .

FU Yucan. Fundamental studies on further developed potentially of high efficiency grinding[D]. Nanjing: Nanjing University of Aeronautics and Astronautics, 1999.

[47] 傅玉灿, 田霖, 徐九华, 等. 一种单颗磨削超高速磨削
实验方法: 中国，201210038994.7[P]. 2012-07-18.

FU Yucan, TIAN Lin, XU Jiuhua, et al. A Method for ultra-high speed grinding with single-grain: China, ZL201210038994.7[P]. 2012-07-18.

[48] 田霖. 基于磨粒有序排布砂轮的高速磨削基础研究[D]. 南京: 南京航空航天大学, 2013.

TIAN Lin. Fundamental research on the high speed grinding with regular abrasive distribution wheel[D]. Nanjing : Nanjing University of Aeronautics and Astronautics, 2013.

[49] 田霖，傅玉灿，杨路，等. 基于速度效应的高温合金高 速超高速磨削成屑过程及磨削力研究 $[\mathrm{J}]$. 机械工程学 报，2013，49(9): 169-177.

TIAN Lin, FU Yucan, YANG Lu, et al. Investigations of the "speed effect" on critical thickness of chip formation and grinding force in high speed and ultra-high speed grinding of superalloy[J]. Journal of Mechanical Engineering, 2013, 49(9): 169-177.

[50] FU Y C, XU H J, XU J H. Optimization design of grinding wheel topography for high efficiency grinding $[\mathrm{J}]$. Journal of Materials Processing Technology, 2002(129): 118-122.

作者简介：傅玉灿(通信作者), 男, 1972 年出生, 教授, 博士研究生导 师。主要研究方向为难加工材料高效精密加工技术。

E-mail: yucanfu@nuaa.edu.cn

田霖，男，1983 年出生，博士。主要研究方向为难加工材料高效磨削技术。 E-mail: tianlinb0905101@foxmail.com 